\title{
Changes in particle size, calcium and phosphate solubilization, and microstructure of rehydrated milk protein concentrates, prepared from partially acidified milk
}

\author{
Hadi Eshpari ${ }^{1,3} \cdot$ Phil S. Tong $^{2} \cdot$ Milena Corredig $^{1}$
}

Received: 17 February 2015 / Revised: 27 September 2015 / Accepted: 12 November 2015 / Published online: 14 January 2016

(C) INRA and Springer-Verlag France 2016

\begin{abstract}
The present work studied the rehydration properties of milk protein concentrates (MPCs), prepared using ultrafiltration (UF) and diafiltration (DF). Milk was acidified to $\mathrm{pH} 6$ with glucono- $\delta$-lactone (GDL) prior to UF to alter the mineral composition of the final concentrates. The particle size distribution and the microstructure of the casein micelles in reconstituted MPCs as well as the partitioning of calcium, phosphate, and proteins between the colloidal and soluble phases were investigated. Reconstituted samples analyzed by electron microscopy showed that, even in partially dissolved particles, the particle surface was porous and similar to its inner portion and had no distinct skin layer. Partial acidification of milk did not have any significant effects on the microstructure; however, it significantly increased the average diameter of the casein micelles for both UF and DF samples and decreased the concentration of total calcium and phosphate. Sodium dodecyl sulfate (SDS)-PAGE analysis of the centrifugal supernatants of reconstituted MPC demonstrated that the amount of soluble caseins present in milk concentrates dramatically increased with acidification, and it further increased after restoring the mineral composition of the serum phase through dialysis against milk. This work contributes to a better understanding of how processing conditions, particularly partial acidification of milk prior to concentration, can alter the composition and physical properties of the caseins and the soluble phase of MPC after rehydration. Such alterations can significantly impact the technological properties of the reconstituted MPC.
\end{abstract}

Milena Corredig

mcorredi@uoguelph.ca

1 Department of Food Science, University of Guelph, Guelph, Ontario N1G 2W1, Canada

2 Dairy Products Technology Center, California Polytechnic State University, San Luis Obispo, CA 93407, USA

3 KraftHeinz R\&D 801 Waukegan Road, Glenview, IL 60025, USA 
Keywords Milk protein concentrate Partial acidification - Rehydration - Casein micelles $\cdot$ Mineral equilibrium $\cdot$ Microstructure

\section{Introduction}

Milk protein concentrates (MPCs) are widely utilized in different food applications such as gels, emulsions, beverages, etc. for their functional and nutritional properties. MPC powders are produced from skim milk by pasteurization, ultrafiltration, diafiltration, evaporation, and spray drying. To take full advantage of their processing functionality, MPC powders must be solubilized in water and form a colloidal dispersion (Gaiani et al. 2007; Martin et al. 2007); however, high protein MPCs such as MPC80 have been reported to exhibit lower solubility than MPC with lower protein concentration or skim milk powder (Mistry and Hassan 1991; Huppertz et al. 2010; Mimouni et al. 2010a).

The existing literature on MPC has been mainly focused on understanding the causes of the increased insolubility (Anema et al. 2005; Gaiani et al. 2006; Havea 2006). Approaches such as modifying the mineral composition or modifying the interactions between the proteins have been successfully practiced to improve solubility of these powders (Carr 2002; Schokker et al. 2011; Sikand et al. 2013; Eshpari et al. 2014). For example, Havea (2006) reported that replacing approximately $30 \%$ of calcium of ultrafiltered skim milk retentate by sodium ions can result in MPC with improved hydration properties.

It is known that the colloidal calcium phosphate in the casein micelles is in equilibrium with the calcium and phosphate of the serum phase, and this balance is fundamental to the structure and function of the micelles (Lucey and Horne 2009). Temperature, $\mathrm{pH}$, and processing conditions will change the distribution of the minerals in milk (Law and Leaver 1998; Le Graët and Gaucheron 1999). Furthermore, concentration of milk using ultrafiltration (UF) results in compositional changes: proteins and minerals associated with them are concentrated in the retentate, while water, lactose, soluble minerals, and non-protein nitrogen are transmitted through the membrane (Mistry and Maubois 2004). It has been shown that a loss of colloidal calcium phosphate during UF and diafiltration causes disruption of the casein micelles ( $\mathrm{Li}$ and Corredig 2014).

Mineral equilibrium also is of importance in determining the rehydration and functional properties of MPC, such as renneting and emulsifying properties (Ferrer et al. 2011; Ye 2011; Sandra and Corredig 2013). Imaging techniques such as scanning electron microscopy (SEM) and transmission electron microcopy (TEM) have been employed to study the microstructure of dairy powders in the dry state (Gaiani et al. 2007; Tamime et al. 2007) or after rehydration (Havea 2006; Mimouni et al. 2010b).

The main objective of the present study was to investigate the effects of partial acidification of milk using glucono- $\delta$-lactone (GDL) before membrane concentration on the size of the casein micelles, distributions of calcium and phosphate in different fractions (colloidal, soluble, and diffusible), and the microstructure of MPC powders after rehydration. It was hypothesized that partial acidification and diafiltration do not have any lasting effects and all the alterations to the partitioning of calcium, phosphate, and caseins between the casein micelles and the serum phase will be reversed upon 
dialysis of samples against skim milk. Furthermore, it was hypothesized that the processing conditions used in this study would have no significant impact on the microstructure of the casein micelles after reconstitution.

\section{Materials and methods}

\subsection{Materials}

Pasteurized skim milk was purchased from Producer's Dairy Foods Inc. (Fresno, CA). Analytical-grade reagents were obtained from Sigma-Aldrich Chemical Co. (St. Louis, MO). GDL was from Roquette America Inc. (Geneva, IL). Upon addition to an aqueous solution, GDL hydrolyzes to gluconic acid, resulting in a controlled decrease of $\mathrm{pH}$. All the solutions were prepared using Ultrapure water (Milli-Q Ultrapure Water Purification Systems, Billerica, MA).

\subsection{Preparation of milk protein concentrates}

The MPC samples were manufactured in duplicate, either by UF (65\% protein MPC65) or by UF followed by diafiltration ( $80 \%$ protein, MPC 80$)$, using pasteurized skim milk $\left(72{ }^{\circ} \mathrm{C}\right.$ for $\left.16 \mathrm{~s}\right)$, at either the native milk $\mathrm{pH}(\sim \mathrm{pH} 6.6)$ or $\mathrm{pH} 6.0$ after addition of GDL, followed by spray drying. GDL (3.25 g.L $\left.{ }^{-1}\right)$ was added to cold milk under continuous stirring and incubated for about $5 \mathrm{~h}$ before membrane filtration. A detailed description of the MPC powder manufacture can be found elsewhere (Eshpari et al. 2014). Four types of MPC powders were manufactured in duplicate: two MPC65, named "UF," and two MPC80, named diafiltration ("DF"), to highlight the differences occurring during the membrane concentration process. The control samples were named "UFC" and "DFC," and the retentates acidified with GDL were named "UFG" and "DFG."

\subsection{Powder rehydration}

Powder samples were reconstituted in ultrapure water (Milli-Q Ultrapure Water Purification Systems, Billerica, MA) to a final protein concentration of $3.2 \%(w / w)$ using a household kitchen blender for $6 \mathrm{~min}$ at high speed. Water was heated to $66{ }^{\circ} \mathrm{C}$ using a heat-stir plate prior to reconstitution. Each reconstituted sample was then divided into two equal portions; one portion was stored in a tightly sealed container at $4{ }^{\circ} \mathrm{C}$ overnight, while the other portion was dialyzed against skim milk at $4{ }^{\circ} \mathrm{C}$ overnight. Dialysis was carried out using an 8000 -Da cutoff membrane (Spectra/Por, Spectrum Laboratories, Inc., Rancho Dominguez, CA, USA) to restore as much as possible the mineral equilibrium state of the reconstituted MPC samples. It was hypothesized that dialysis will reverse any alterations caused by the acidification and the membrane concentration treatments. Dialysis would indeed reestablish a serum composition as similar as possible to that of the original skim milk, to make possible a comparison of the casein micelle processing behavior across all treatments. The samples were named "UFC-D and UFG-D" and "DFC-D and DFG-D" after dialysis. 


\subsection{Light scattering measurements}

Particle size distribution of the MPC samples reconstituted as described above was determined by integrated light scattering (Mastersizer 2000, Malvern Instruments, Southborough, MA). The volume-weighted mean particle diameter $\left(\mathrm{d}_{4,3}\right)$ was recorded. A few drops of reconstituted MPC dispersions (temperature adjusted to $25^{\circ} \mathrm{C}$ ) were added into the small-dispersion unit to reach an obscuration level of 10 to 20 (dilution factor in water was approximately $10^{-3}$ ) while stirring. The refractive indexes used in particle size distribution calculations were 1.39 for the casein micelles (Alexander et al. 2002) and 1.33 for water.

The apparent diameter of the casein micelles was measured using a dynamic light scattering (DLS) instrument (Zetasizer, Nano-ZS, Malvern Instruments, Malvern, UK) by diluting each sample in skim milk permeate (temperature adjusted to $25^{\circ} \mathrm{C}$ ) about 1000 times, to avoid multiple scattering. Fresh skim milk permeate was prepared by UF of skim milk (Crown Dairy, Guelph, ON, Canada) using an OPTISEP ${ }^{\mathbb{B}}$ Filter module (Smartflow Technologies, Apex, NC, USA) with 10-kDa molecular mass cutoff at $25{ }^{\circ} \mathrm{C}$. Sodium azide was added at $0.02 \%(w / v)$ to the obtained permeate, before refrigerating it at $4{ }^{\circ} \mathrm{C}$.

\subsection{Protein composition of the soluble fraction}

The protein composition of the soluble fraction of the MPC samples reconstituted at $3.2 \%$ equal protein (as described in Sect. 2), and a pasteurized skim milk sample (as control) was analyzed using sodium dodecyl sulfate (SDS)-PAGE both before and after dialysis. The centrifugal supernatant obtained from centrifugation at $61,000 \times g$ for $30 \mathrm{~min}$ at $25^{\circ} \mathrm{C}$ was mixed $(1: 1$ ratio) with electrophoresis buffer, containing $1 \mathrm{M}$ Tris-HCl buffer, $\mathrm{pH}$ 6.8, 75\% glycerol, 10\% SDS, 2-mercaptoethanol, and 1\% bromophenol blue. The solution was heated and mixed at $95{ }^{\circ} \mathrm{C}$ for $5 \mathrm{~min}$ using a thermomixer (model 5436; Eppendorf, Hauppauge, NY, USA). Samples were cooled to room temperature, and $10 \mu \mathrm{L}$ of each sample was then loaded onto the gels. SDSPAGE was carried out in a vertical slab gel of $1.5-\mathrm{mm}$ thickness with $15 \%$ acrylamide resolving gel and $4 \%$ stacking gel in a Bio-Rad mini-protein electrophoresis system (Bio-Rad Laboratories, Hercules, CA, USA) at a constant voltage of $175 \mathrm{~V}$. The gels were then treated with Coomassie blue R-250 for $45 \mathrm{~min}$ (Bio-Rad) and destained with $45 \%$ Milli-Q water, $45 \%$ methanol, and $10 \%$ glacial acetic acid for $1 \mathrm{~h}$ and then with the same solution diluted 1:1 in water overnight. The gels were scanned using a BioRad Gel Doc EZ Imager (Bio-Rad Power Pac HC, Hercules, CA) equipped with Image Lab 3.0 software (Bio-Rad Power Pac HC, Hercules, CA).

\subsection{Calcium and phosphate determination}

The concentration of inorganic calcium and phosphate in different fractions (total, soluble, and non-protein bound) of reconstituted powders (equal 3.2\% protein) as well as a skim milk sample (for comparison) was measured using ion chromatography. The calcium and phosphate measured in the supernatant of reconstituted powders after centrifugation at $65,000 \times g$ for $1 \mathrm{~h}$ at $25^{\circ} \mathrm{C}$ (see above) were referred to as soluble calcium and phosphate. It is important to note that, this non-sedimentable soluble 
fraction contained both protein-bound and protein-free calcium and phosphate. To separate the protein-free fraction of calcium and phosphate, in a different experiment, the reconstituted powders were filtered using a Prep/ScaleTM-TEF $1 \mathrm{ft} 2$ cartridge UF unit (10-kDa cutoff regenerated cellulose, Millipore, Ontario, Canada). The resulting permeate contained the protein-free fraction of both calcium and phosphate, also referred to as permeable or diffusible calcium and phosphate.

It is recognized that the amount of soluble minerals present plays a major role in determining the functional properties of the milk proteins including rennetability, thermal stability, and surface and rheological properties (De la Fuente 1998); hence, it was considered necessary in this work to measure the concentration of both soluble and permeable calcium and phosphate.

The amount of calcium was estimated as reported in the literature (Rahimi-Yazdi et al. 2010). Analyses were carried out in duplicate for each sample by an Advanced Compact IC (Metrohm AG, Zurich, Switzerland), using a silica gel column (Metrosep C2 150/40 packed with $7 \mathrm{~mm}$ silica gel; Metrohm AG) at $30{ }^{\circ} \mathrm{C}$. To measure the amount of total and soluble phosphate, $1 \mathrm{~mL}$ of each fraction was transferred to a $5-\mathrm{mL}$ Pyrex test tube (Corning Incorporated Life Sciences, MA, USA) and dry-ashed by initial heating at $100{ }^{\circ} \mathrm{C}$ overnight to dry the samples, followed by further heating at $500{ }^{\circ} \mathrm{C}$ for $6 \mathrm{~h}$ to remove the organic matter. The dry ashing was conducted in an Isotemp muffle furnace (Fisher Scientific, MA, USA). The obtained ashes were dissolved in $1 \mathrm{~mL}$ of $1 \mathrm{~mol} . \mathrm{L}^{-1} \mathrm{HNO}_{3}$ to be injected into the HPLC. No dry ashing was necessary in sample preparation for the permeable phosphate analysis, as the sample did not contain any bound proteins at this point. An anion column (Metrosep A Supp5-150/4.0, Metrohm) packed with $5 \mu \mathrm{m}$ polyvinyl alcohol with quaternary ammonium groups was employed to measure the phosphate. Sodium hydrogen carbonate and sodium carbonate solutions were used to prepare mobile phase (1.0 $\mathrm{mM}$ sodium carbonate and $4 \mathrm{mM}$ sodium hydroxide). Samples were eluted at a flow rate of $0.5 \mathrm{~mL} \cdot \mathrm{min}^{-1}$.

\subsection{Microstructure of reconstituted milk protein concentrate powders}

\subsubsection{Cryogenic transmission electron microscopy}

Cryogenic TEM (cryo-TEM) was employed to provide information on the internal structure of the particles in the rehydrated MPC samples. The sample $(4 \mu \mathrm{L})$ was pipetted onto a quantifoil (Quantifoil Micro, Jena, Germany) grid with 2- $\mu$ m holes. The excess sample was blotted off in a vitrobot (FEI) (Eindhoven, Holland) and immediately plunged into liquid ethane held at liquid nitrogen temperature. The specimen was transferred under liquid nitrogen to a Gatan 626 Cryo holder (Warrendale, PA, USA) and viewed at $-176{ }^{\circ} \mathrm{C}$ in the Tecnai G2 F20 TEM (Eindhoven, Holland). Images were recorded with a Gatan $4 \mathrm{~K}$ bottom mount CCD camera using the Gatan Digital Micrograph software.

\subsubsection{Scanning electron microscopy}

The surface microstructure of the rehydrated MPC powders was determined using scanning electron microscopy (SEM) according to the method described by Mimouni 
et al. (2010a, b). Samples were dried using $\mathrm{CO}_{2}$ in a critical point dryer and sputtercoated for 2 min with a layer of 5-nm thickness of Au/Pd (ratio 60:40; Emitech K550, Ashford, Kent, UK) and stored in a desiccator at room temperature until imaging. The imaging was conducted using a XHR (extreme high resolution) scanning electron microscope, FEI Magellan 400 (FEI, Tokyo, Japan), operating at 1.00 and $2.00 \mathrm{kV}$, at the Canadian Centre for Electron Microscopy of McMaster University (Hamilton, Canada).

\subsection{Statistical analysis}

The sample treatments and analyses in the present study were performed in duplicate. Values were means of replicate determinations, and the differences between the means of the treatments were compared by one-way ANOVA at a significance level of $P<0.05$. The statistical analysis was conducted using the GLM command in Minitab (v.16.1, Minitab Inc., State College, PA). Differences between the treatments were tested using Tukey's honestly significant difference (HSD) intervals with $\alpha=0.05$.

\section{Results and discussion}

\subsection{Particle size distribution}

The particle size distribution of the casein micelles in the reconstituted powders was determined using integrated light scattering, as shown in Fig. 1. Powders were reconstituted with water at $66^{\circ} \mathrm{C}$, using a blender for $6 \mathrm{~min}$. The samples were analyzed immediately after reconstitution, as well as after overnight storage at $4{ }^{\circ} \mathrm{C}$ or after dialysis against skim milk at $4{ }^{\circ} \mathrm{C}$. There were no statistically

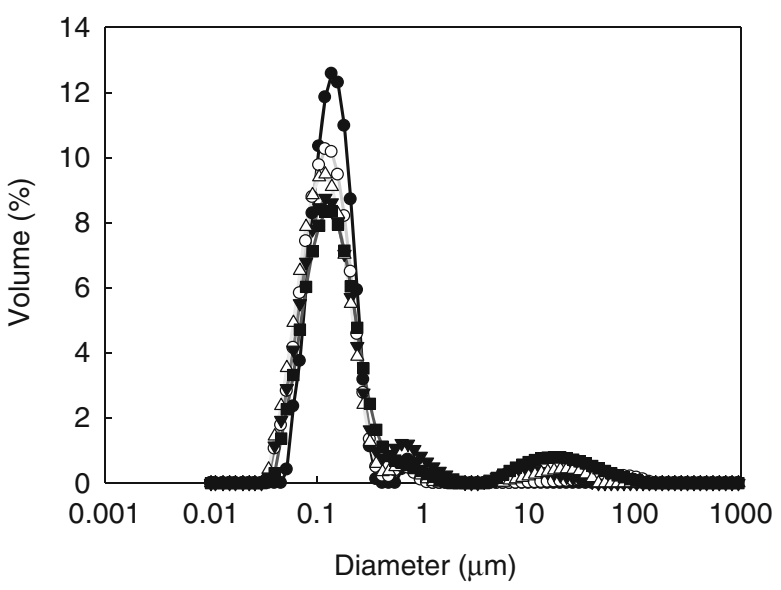

Fig. 1 Particle size distribution of reconstituted concentrates. Fresh, pasteurized skim milk (closed circle); reconstituted MPC65 (control, UFC, open circle) (acidified, UFG, closed inverted triangle); reconstituted MPC80 (control, DFC, open triangle) (acidified, DFG, closed square gray broken line). Graph is representative of two replicate samples 
significant differences in the particle size distribution of all the reconstituted MPC powders. All dispersions showed a main peak appearing between 0.01and $1-\mu \mathrm{m}$ diameter, corresponding to that of native casein micelles. However, the powders also showed the presence of larger particles between 10 and $100 \mu \mathrm{m}$. Similar size distributions were observed also after dialysis (data not shown). These results indicated that the rehydration at $66{ }^{\circ} \mathrm{C}$ was effective in regaining the original distribution of casein micelles. The distributions were comparable to those of skim milk (also shown in Fig. 1), in agreement with the previous reports (Ferrer et al. 2008).

The apparent diameter of the casein micelles in reconstituted milk concentrates was also measured by DLS (Table 1). The apparent diameter of casein micelles in control skim milk was $169.2 \pm 0.9 \mathrm{~nm}$. No significant changes of diameter were detected in reconstituted control MPC samples (UFC and DFC) compared to skim milk, in agreement with other research reported in the literature (Beliciu and Moraru 2009,). However, the acidified samples (UFG and DFG) exhibited a significant increase in the apparent diameter of the reconstituted casein micelles. This could be due to an increase in the attractive forces between proteins. The DFG sample showed the largest average diameter, indicating a significant effect of DF in these retentates. However, such changes in diameter associated with the acidification have been previously attributed to depletion of colloidal calcium phosphate (CCP) from the casein micelles, leading to a decrease of intramicellar protein interactions, an increase in charge repulsion, and an increase in the water adsorption, resulting in the increase in voluminosity. This would support the findings of Gaucheron (2005), who suggested an increased of casein micelle voluminosity with a change in the mineral equilibrium in milk. A larger apparent diameter of casein micelles in UFG and DFG compared to control samples was also shown for reconstituted samples after dialysis against milk. The change in size was not reversible after restoring the mineral composition of the serum phase.

Table 1 Apparent diameter of casein micelles after reconstitution, as measured by dynamic light scattering

\begin{tabular}{ll}
\hline Sample & Diameter $(\mathrm{nm})$ \\
\hline SM & $169.2 \pm 0.9^{\mathrm{a}}$ \\
UFC & $169.5 \pm 0.6^{\mathrm{a}}$ \\
UFG & $197.5 \pm 0.9^{\mathrm{b}}$ \\
DFC & $177.4 \pm 2.0^{\mathrm{ab}}$ \\
DFG & $207.5 \pm 8.6^{\mathrm{c}}$ \\
UFC-D & $173.9 \pm 0.9^{\mathrm{a}}$ \\
UFG-D & $202.4 \pm 1.3^{\mathrm{b}}$ \\
DFC-D & $178.8 \pm 1.7^{\mathrm{ab}}$ \\
DFG-D & $212.7 \pm 8.1^{\mathrm{c}}$
\end{tabular}

Data are average of two independent experiments. The letters indicate significant differences across treatments by Tukey's honestly significant difference procedure, for $p<0.05$. Skim milk (SM), UFC and DFC, and UFG and DFG were reconstituted and measured after overnight storage at $4^{\circ} \mathrm{C}$, as well as after dialysis against milk (-D) at $4{ }^{\circ} \mathrm{C}$ overnight 
It was concluded that the ionic distribution was not the only cause of the larger average size, but structural changes may have occurred to the structure of the micelles, in the acidified MPC samples.

\subsection{Protein composition of the soluble fraction}

The protein composition in the centrifugal supernatants was analyzed by SDS-PAGE as shown in Fig. 2. All samples were reconstituted at the same protein concentration, and a higher intensity of band would indicate an increase in the amount of protein recovered in the supernatant. The soluble fractions recovered in the reconstituted MPC were also compared with those of pasteurized skim milk (Fig. 2, lane 1). A higher concentration of soluble caseins was present in the supernatant of reconstituted MPC samples (lanes 2 to 9) compared to skim milk (lane 1) despite the equal initial protein content $3.2 \%(\mathrm{w} /$ $w$ ). It has been previously demonstrated that after membrane concentration, there is an increase in soluble caseins in both fresh or reconstituted retentates (Ferrer et al. 2011; Sandra and Corredig 2013). The soluble fraction varies depending on the concentration and the presence of DF (Li et al. 2015). The results shown in Fig. 2 further demonstrate that the amount of soluble caseins increased in the reconstituted MPCs that were concentrated at lower $\mathrm{pH}$ (Fig. 2, lanes 4, 5, 8, and 9), compared to control MPC (Fig. 2, lanes 2, 3, 6, and 7).

The increase in the amount of casein proteins in the centrifugal supernatant of the reconstituted MPC made from the acidified milk was much larger after dialysis against milk compared to the increase occurring in control reconstituted MPC. Dialysis caused a reequilibration of the serum phase composition, with values of calcium and phosphate similar to those present in the original milk. The increase of disruption of the casein micelles with reequilibration was consistent with what previously reported (Sandra and Corredig 2013). Restoring the mineral composition of the serum phase of the reconstituted MPC caused an increase in soluble calcium and phosphate, and this leads to further solubilization of the proteins. This behavior was dependent on $\mathrm{pH}$ during membrane concentration.

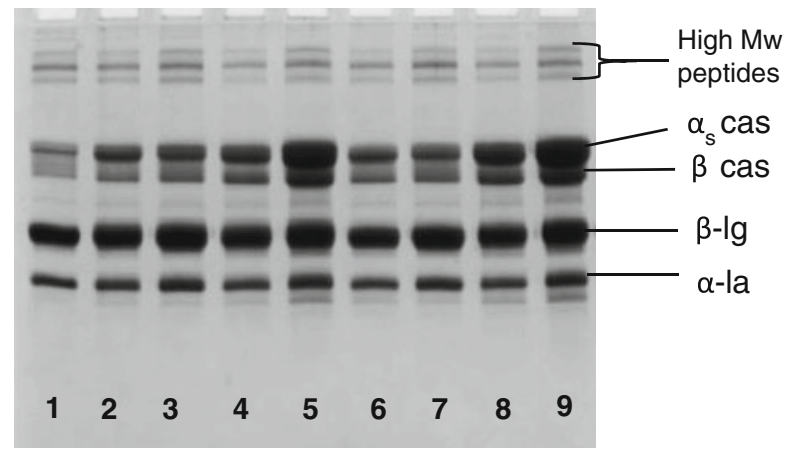

Fig. 2 SDS-PAGE of centrifugal supernatants $(10 \mu \mathrm{L})$ of pasteurized skim milk (lanes 1) and reconstituted MPC (lanes 2-9). All samples were reconstituted at 3.2\% (w/w) protein. UF control: UFC (lane 2) and UFC-D (lane 3); UF acidified: UFG (lane 4) and UFG-D (lane 5); DF control: DFC (lane 6) and DFC-D (lane 7); DF acidified: DFG (lane 8) and DFG-D (lane 9). Results are representative of two replicate runs 


\subsection{Calcium and phosphate distributions in the micellar, soluble, and diffusible phases of reconstituted milk protein concentrate before and after dialysis against skim milk}

Figure 3 illustrates the concentration of total, soluble, and permeable calcium (Fig. 3a, b) and phosphate (Fig. 3c, d) in MPC samples reconstituted at equal 3.2\% $(w / w)$ protein compared to pasteurized skim milk. All MPC samples showed a significantly lower total calcium content compared to fresh skim milk, regardless of treatments. As expected, amongst reconstituted MPC, the total calcium content was the lowest in the acidified samples after diafiltration (DFG) and the highest in the UF control (UFC) (Fig. 3a). The acidification to $\mathrm{pH} 6$ also showed a significant increase of the soluble and permeable calcium contents in the ultrafiltered samples (UFG > UFC), while after DF, there was a significant decrease of the permeable calcium in the acidified samples (DFG $<$ UFG). This was due to the release of calcium during acidification and the presence of a higher amount of permeable calcium in UFG, which was further transmitted through the membrane during DF.

The same samples were also analyzed after dialysis against skim milk (Fig. 3b). In this case, the total calcium content was restored in the case of UF and DF control samples (UFC-D and DFC-D). The total calcium content increased also in the acidified MPC samples, although it remained at a significantly lower level compared to the total calcium content of skim milk and the dialyzed MPC control. With regard to soluble and

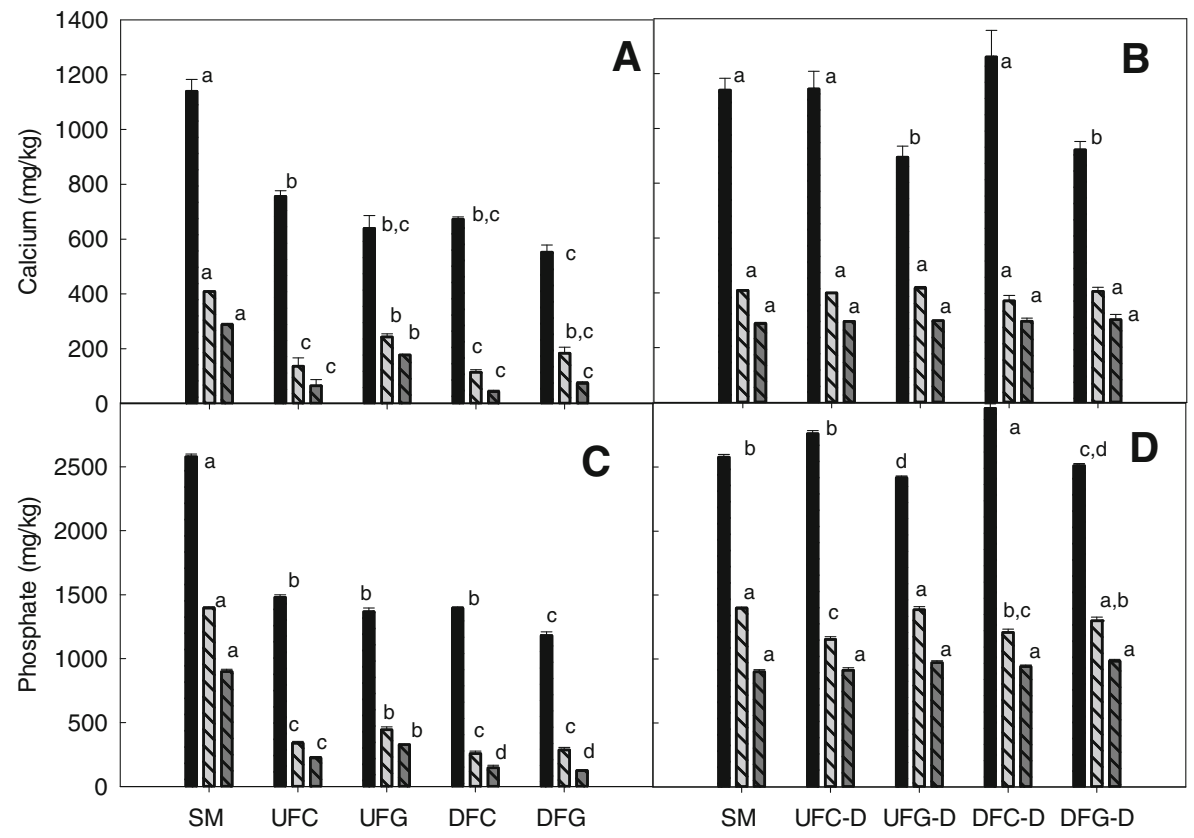

Fig. 3 Total (black bars), soluble (gray bars), and permeable (dark gray bars) calcium (A, B) and phosphate $(\mathbf{C}, \mathbf{D})$ for skim milk control (SM) and various MPC resuspended to 3.2\% (w/w) protein, before (A, C) or after (B, D) dialysis against skim milk. UF control (UFC, UFC-D), UF acidified (UFG, UFG-D), DF control (DFC, DFC-D), and DF acidified (DFG, DFG-D). Data are average of two independant experiments. Treatments with different letters indicate significant differences. Error bars represent standard deviations 
permeable calcium, dialysis against milk restored their concentrations in all MPC samples (regardless of treatments). All reconstituted MPC samples, after dialysis, including those processed with GDL, had a comparable concentration of soluble and permeable calcium. However, it is important to note that UFG and UFG-D showed a lower level of colloidal calcium (as calculated by difference between total and soluble calcium) associated to the casein micelles.

Figure $3 \mathrm{c}, \mathrm{d}$ also summarizes the concentration of total, soluble, and permeable phosphate in the reconstituted MPC samples compared to pasteurized skim milk. After reconstitution (Fig. 3c), all samples exhibited significantly lower total phosphate compared to skim milk, similarly to calcium (Fig. 3a), indicating a significant decrease for all MPC samples, regardless of treatments. Amongst the reconstituted MPC, the total phosphate content was the lowest in the acidified samples after diafiltration (DFG), with the other treatments not showing any statistically significant differences. Partial acidification of UF retentates showed a significant increase for both soluble and permeable phosphate. After DF, the soluble phosphate was comparable to control UF, but the permeable phosphate significantly decreased (Fig. 3c). After dialysis against skim milk (Fig. 3d), the total phosphate content increased in all MPC samples. In control MPC samples, after DF, the concentration of total phosphate exceeded that found in skim milk, with diafiltered control (DFC-D) being significantly higher than UF control (UFC-D). This may indicate an increase in the unsedimentable phosphate after dialysis. The total phosphate content of the acidified sample after dialysis was lower or similar to that of control skim milk. A similar trend was also observed in the acidified samples than in control MPC; after dialysis, the DF samples showed a higher total phosphate concentration than the UF samples. It is important to note that all these samples had a comparable protein concentration. Soluble phosphate was also restored in acidified samples after dialysis. Furthermore, dialysis restored the concentrations of permeable phosphate in all MPC samples to the same level of skim milk, regardless of the treatments.

\subsection{Microstructure of casein micelles after reconstitution}

Figure 4 illustrates cryo-transmission electron microscope (TEM) images of casein micelles in reconstituted MPC samples. All samples, regardless of their treatments, exhibited an even distribution of small electron-dense regions throughout the micellar structure. The existence of such structural features in casein micelles has also been previously reported; the electron-dense regions have been attributed to the calcium phosphate domains within the casein micelles (Marchin et al. 2007). The cryo-TEM observations demonstrated that the partial acidification of milk to $\mathrm{pH} 6$ prior to concentration by UF or DF did not show any substantial changes in the internal microstructural appearance of the casein micelles. No differences were observed in the electron density of the acidified micelles. The acidified micelles exhibited an intact boundary appearing as well defined as in the control micelles. These observations are different from those previously reported for casein micelles acidified at much lower $\mathrm{pH}$ values (Marchin et al. 2007). Indeed, while at pH 5.2, most of the colloidal calcium has 

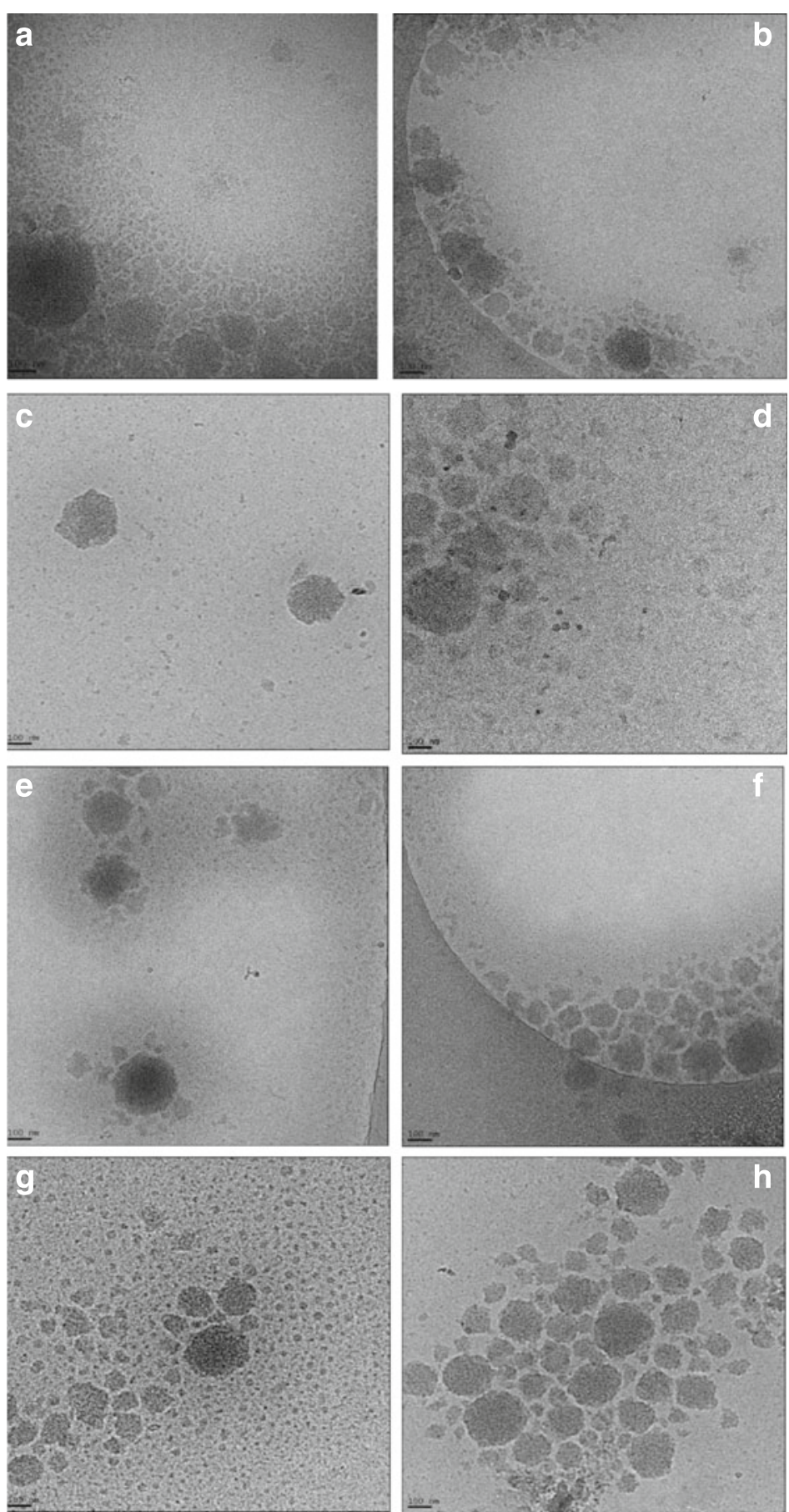

Fig. 4 Cryo-TEM micrographs of MPC samples reconstituted at 3.2\% (w/w) protein. Control: UFC (a, b) and DFC (e, f); acidified: UFG (c, d) and DFG $(\mathbf{g}, \mathbf{h})$. Scale bar $=100 \mathrm{~nm}$ 
been removed from the casein micelles; in the present study, calcium phosphate nanoparticles are still present, as also shown in Fig. 3.

The TEM observations summarized in Fig. 4 demonstrated that reconstitution of the MPC did not affect the structure of the micelles. However, it may appear that DF caused some disruption of the micelles, as shown by the presence of smaller aggregates in the DF sample. Furthermore, in all the samples, there was no film around the large clusters of micelles. The presence of aggregates around protein particles during reconstitution has been reported before (Mimouni et al. 2010b); however, this film was absent under the reconstitution conditions used in this work.

The microstructure of the reconstituted samples was also observed using scanning electron microscopy (SEM). Figure 5 depicts images of the casein micelles in control skim milk. The particles showed a raspberry-like surface structure typical of casein micelles as previously demonstrated (Dalgleish et al. 2004). Figure 6 illustrates the field emission scanning electron microscope (SEM) images of the reconstituted MPC powders. It is worth noting the presence of some partially dissolved larger particles (diameter of 4-6 $\mu \mathrm{m}$ ) in the samples across all treatments. All samples showed the presence of particles with a size range typical of the casein micelles in milk and average diameter of about $200 \mathrm{~nm}$ (Holt et al. 2003). These smaller particles represented the fully dissolved larger portion of each MPC powder. These results are in perfect agreement with the particle size distribution profiles of the MPC samples (Fig. 1). Moreover, the surface microstructure of the particles, even the partially dissolved larger ones, was as porous as their inner portion (Fig. 6c, g, f), without any distinct surface layer, referred to as skin in the recent MPC literature (Mimouni et al. 2010b). It was observed that the casein micelles were closely packed and linked to each other in a network which is gradually loosened as a result of water penetration during the rehydration process (Fig. 6b, d-f).

\section{Conclusions}

Membrane technologies are often used to standardize milk, and it is important to better understand the changes that may occur to the structure and function of the casein micelles after those unit operations. The solubility of the casein micelles after reconstitution was evaluated. Partial acidification significantly increased the average apparent diameter of the reconstituted casein micelles in both UF and DF samples.
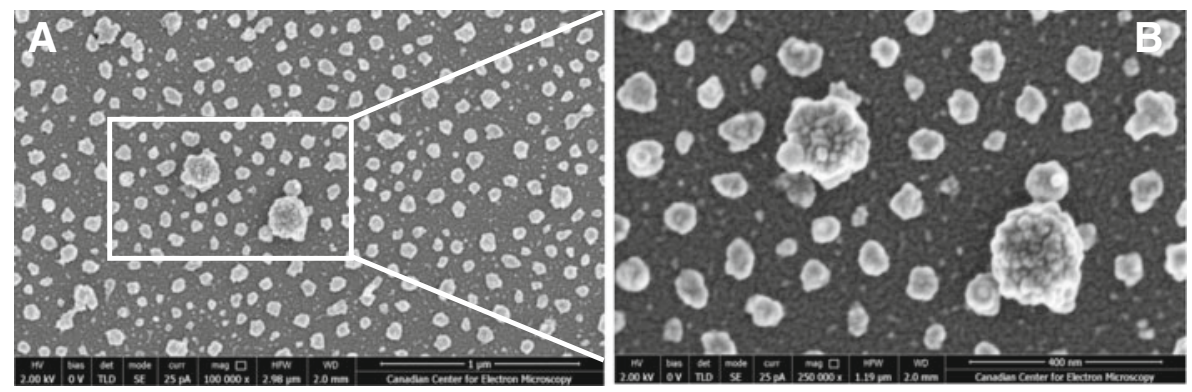

Fig. 5 SEM images of casein micelles in skim milk (scale bar $1 \mu \mathrm{m}$ (a) and $400 \mathrm{~nm}$ (b)) 

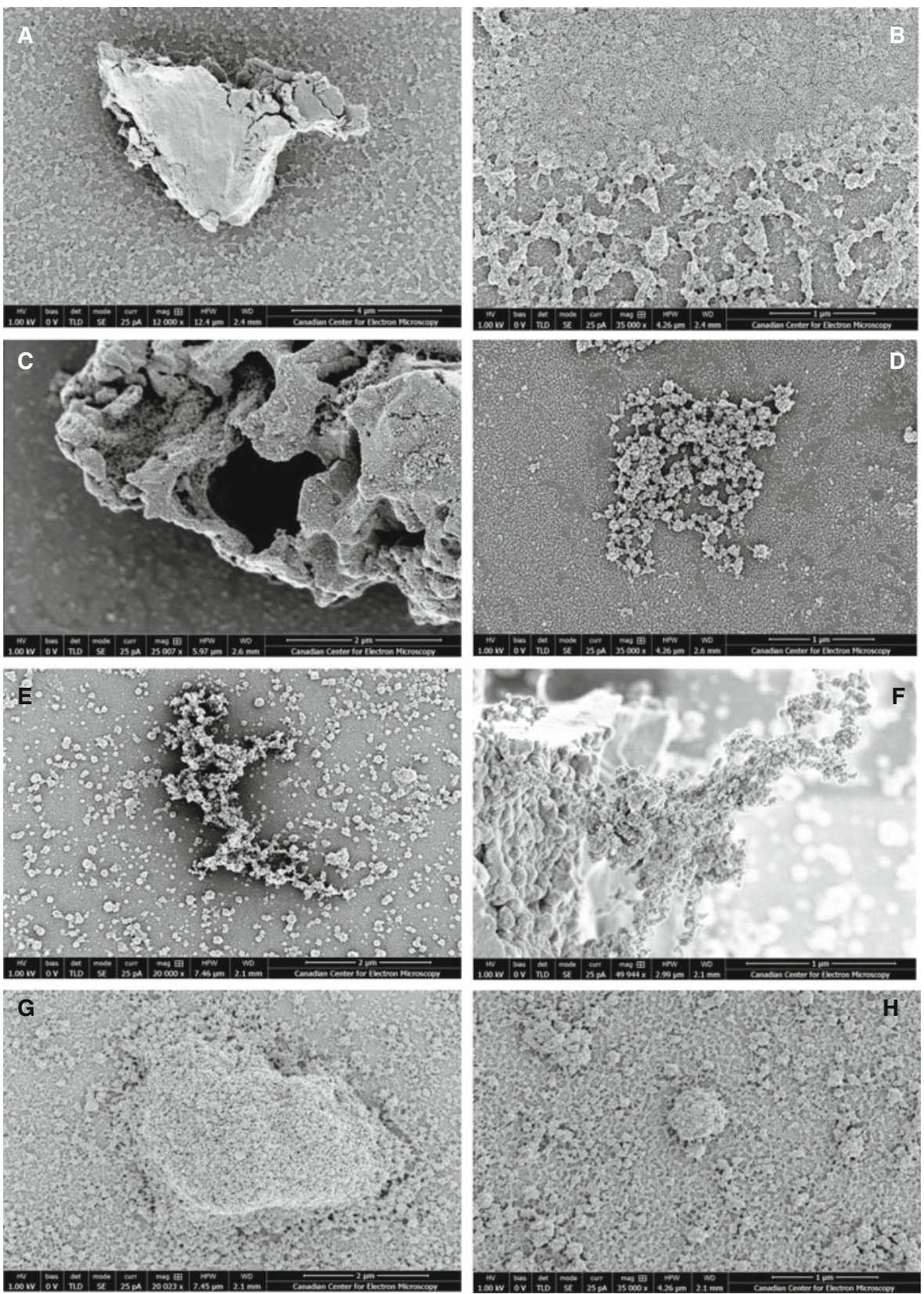

Fig. 6 SEM images of MPC samples reconstituted to 3.2\% (w/w) protein. UFC (a, scale bar $4 \mu \mathrm{m}$; b, scale bar $1 \mu \mathrm{m})$; UFG (c, scale bar $2 \mu \mathrm{m}$; d, scale bar $1 \mu \mathrm{m})$; DFC (e, scale bar $2 \mu \mathrm{m} ; \mathbf{f}$, scale bar $1 \mu \mathrm{m})$; DFG (g, scale bar $2 \mu \mathrm{m} ; \mathbf{h}$, scale bar $1 \mu \mathrm{m})$

Significant differences were also observed in the partitioning of calcium, phosphate, and proteins between the colloidal and the soluble phase. In general, acidification decreased the concentrations of total calcium and phosphate but increased the 
concentrations of soluble and permeable calcium and phosphate. Acidification to $\mathrm{pH} 6$ before membrane filtration decreased the amount of colloidal calcium present in the micelles and increased the amount of soluble caseins. The disruption of the casein micelles increased after dialysis of the reconstituted samples against skim milk. By dialysis, it was possible to a great extent to reverse the changes in the mineral equilibrium caused by the partial acidification and DF, and this caused further disruption of the casein micelles. In addition, there was a significantly higher level of soluble caseins in the rehydrated MPC samples, regardless of their acidification or DF status, indicating a partial dissociation of casein micelles as a result of MPC manufacturing process. The amount of soluble caseins recovered in the supernatant was higher in the acidified samples compared to the controls and after dialysis against skim milk.

Despite all the changes observed in the calcium and phosphate distributions and the increase in both the apparent diameter and the amount of soluble caseins present in the reconstituted MPC made from acidified milk, no differences were observed in the internal and surface microstructure of the casein micelles. Furthermore, there was no evidence of a film surrounding the protein particles during reconstitution. It was concluded that although pre-acidification of milk to $\mathrm{pH} 6$ in the process of the MPC manufacture caused some lasting modifications to the casein micelles including an increase of the apparent diameter, partial solubilization of CCP and partial dissociation of the caseins, the micelles maintained their shape and colloidal stability. The MPC made up of such compositionally modified, swollen casein particles would have unique technological properties (e.g., rennetability, cheese making, and thermal stability) which are well worth further investigation.

Acknowledgments This project was partly funded by the Ontario Dairy Council (Ottawa, ON, Canada) and the Natural Science and Engineering Council of Canada (Ottawa, ON, Canada). Support was also provided by California Dairy Research Foundation (Davis, CA) and Dairy Research Incorporated (Rosemont, IL). Special thanks to Sean Vink (Dairy Products Tech. Center, California Polytechnic State University, San Luis Obispo) for assistance in the powder manufacture. Dr. Alexandra Smith (Food Science Department, University of Guelph) is greatly thanked for her assistance in the sample preparation for SEM.

\section{References}

Alexander M, Rojas-Ochoa LF, Leser M, Schurtenberger P (2002) Structure, dynamics, and optical properties of concentrated milk suspensions: an analogy to hard-sphere liquids. J Colloid Interface Sci 253:35-46

Anema SG, Pinder DN, Hunter RJ, Hemar Y (2005) Effects of storage temperature on the solubility of milk protein concentrate (MPC85). Food Hydrocoll 20:386-393

Beliciu CM, Moraru CI (2009) Effect of solvent and temperature on the size distribution of casein micelles measured by dynamic light scattering. J Dairy Sci 92:1829-1839

Carr AJ (2002) Monovalent salt enhances solubility of milk protein concentrate. New Zealand Dairy Board, assignee. International Patent No. WO02/096208.

Dalgleish DG, Spagnuolo PA, Goff HD (2004) A possible structure of the casein micelle based on highresolution field-emission scanning electron microscopy. Int Dairy J 14:1025-1031

De la Fuente MA (1998) Changes in the mineral balance of milk submitted to technological treatments. Trends Food Sci Technol 9:281-288

Eshpari H, Tong PS, Corredig M (2014) Changes in the physical properties, solubility, and heat stability of milk protein concentrates prepared from partially acidified milk. J Dairy Sci 97(12):7394-7401

Ferrer M, Hill AR, Corredig M (2008) Rheological properties of rennet gels containing milk protein concentrates. J Dairy Sci 91(3):959-969 
Ferrer M, Alexander M, Corredig M (2011) Does ultrafiltration have a lasting effect on the physico-chemical properties of the casein micelles? Dairy Sci Technol 91(2):151-170

Gaiani C, Scher J, Schuck P, Hardy J, Desobry S, Banon S (2006) The dissolution behavior of native phosphocaseinate as a function of concentration and temperature using a rheological approach. Int Dairy J 16:1427-1434

Gaiani C, Schuck P, Scher J, Desobry S, Banon S (2007) Dairy powder rehydration: influence of protein state incorporation mode, and agglomeration. J Dairy Sci 90:570-581

Gaucheron F (2005) The minerals of milk. Reprod Nutr Dev 45:473-483

Havea P (2006) Protein interactions in milk protein concentrate powders. Int Dairy J 16:415-422

Holt C, De Kruif CG, Tuinier R, Timmins PA (2003) Substructure of bovine casein micelles by small-angle Xray and neutron scattering. Colloids Surf A Physicochem Eng Asp 213:275-284

Huppertz T, Alting A, Slangen C, Floris R (2010) Milk protein concentrate functionality (oral presentation). http:/www.wds2010.com/delegates/presentations/09tue/05-Session3_2-Thom\%20Huppertz.pdf. Accessed Sept 92012

Law AJR, Leaver J (1998) Effects of acidification and storage of milk on dissociation of bovine casein micelles. J Agric Food Chem 46:5008-5016

Le Graët Y, Gaucheron F (1999) pH-induced solubilization of minerals from casein micelles: influence of casein concentration and ionic strength. J Dairy Res 66:215-224

Li Y, Corredig M (2014) Calcium release from milk concentrated by ultrafiltration and diafiltration. J Dairy Sci 97(9):5294-5302

Li Y, Dalgleish DG, Corredig M (2015) Influence of heating treatment and membrane concentration on the formation of soluble aggregates. Food Res Int 76:309-316

Lucey JA, Horne DS (2009) Milk salts: technological significance. In Advanced dairy chemistry_volume 3: lactose, water, salts and minor constituents. 351- 390 (Ed., P. F. Fox), Springer New York

Marchin S, Putaux JL, Pignon F, Leonil J (2007) Effects of the environmental factors on the casein micelle structure studied by cryo transmission electron microscopy and small-angle $\mathrm{X}$-ray scattering/ultrasmallangle x-ray scattering. J Chem Phys 126:045101

Martin GJO, Williams RPW, Dunstan DE (2007) Comparison of casein micelles in raw and reconstituted skim milk. J Dairy Sci 90:4543-4551

Mimouni A, Deeth HC, Whittaker AK, Gidley MJ, Bhandari BR (2010a) Rehydration of high-proteincontaining dairy powder: slow- and fast-dissolving components and storage effects. Dairy Sci Technol 90(2):335-344

Mimouni A, Deeth HC, Whittaker AK, Gidley MJ, Bhandari BR (2010b) Investigation of the microstructure of milk protein concentrate powders during rehydration: alterations during storage. J Dairy Sci 93(2):463472

Mistry V, Hassan H (1991) Delactosed high milk protein powder. 2 Physical and functional properties. J Dairy Sci $74: 3716-3723$

Mistry V, Maubois JL (2004) Application of membrane separation technology to cheese production, vol 1, 3rd edn, Cheese chemistry, physics, and microbiology. Elsevier Academic Press, London, UK, pp 261-286

Rahimi-Yazdi S, Ferrer MA, Corredig M (2010) Nonsuppressed ion chromatographic determination of total calcium in milk. J Dairy Sci 93:1788-1793

Sandra S, Corredig M (2013) Rennet induced gelation of reconstituted milk protein concentrates: the role of calcium and soluble proteins during reconstitution. Int Dairy J 29:68-74

Schokker E, Church J, Mata J, Gilbert E, Puvanenthiran A, Udabage P (2011) Reconstitution properties of micellar casein powder: effects of composition and storage. Int. Dairy J 21:877-886

Sikand V, Tong PS, Walker J (2013) Effect of adding salt during the diafiltration step of milk protein concentrate powder manufacture on mineral and soluble protein composition. J Dairy Sci Technol 93: 401-413

Tamime AY, Robinson RK, Michel M (2007) Microstructure of concentrated and dried milk products. In: Tamime AY (ed) Structure of dairy products. Blackwell Publishing Ltd, Oxford, UK, pp 104-133

Ye A (2011) Functional properties of milk protein concentrates: emulsifying properties, adsorption and stability of emulsions. Int Dairy J 21(1):14-20 